\title{
Adult-onset Krabbe disease in two generations of a Chinese family
}

\author{
Tongxia Zhang ${ }^{1}$, Chuanzhu Yan ${ }^{1,2,3}$, Kunqian Ji ${ }^{1}$, Pengfei Lin $^{1}$, Lingyi Chi $^{2,4}$, Xiuhe Zhao ${ }^{1}$, Yuying Zhao ${ }^{1}$ \\ ${ }^{1}$ Research Institute of Neuromuscular and Neurodegenerative Diseases and Department of Neurology, ${ }^{2}$ Brain Science Research Institute, Qilu \\ Hospital, Shandong University, Jinan 250012, China; ${ }^{3}$ Mitochondrial Medicine Laboratory, Qilu Hospital (Qingdao), Shandong University, Qingdao \\ 266035, China; ${ }^{4}$ Department of Neurosurgery, Qilu Hospital, Shandong University, Jinan 250012, China \\ Contributions: Conception and design: Y Zhao, C Yan; (II) Administrative support: C Yan; (III) Provision of study materials or patients: Y Zhao, T \\ Zhang; (IV) Collection and assembly of data: T Zhang, K Ji, P Lin, L Chi, X Zhao; (V) Data analysis and interpretation: T Zhang, K Ji, P Lin, L \\ Chi, X Zhao; (VI) Manuscript writing: All authors; (VII) Final approval of manuscript: All authors. \\ Correspondence to: Dr. Yuying Zhao. Research Institute of Neuromuscular and Neurodegenerative Diseases and Department of Neurology, Qilu \\ Hospital, Shandong University, Jinan 250012, China. Email: zyy72@126.com.
}

\begin{abstract}
Background: Krabbe disease (KD) is a rare autosomal recessive lysosomal storage disorder caused by deficiency of the galactocerebrosidase (GALC) enzyme. The adult-onset KD is infrequent, and often presenting with slowly progressive spastic paraplegia. Herein, we describe a two-generation concomitant Chinese pedigree of adult-onset KD in which the proband presented with acute hemiplegia at onset.

Methods: We collected the clinical and neuroimaging data of the pedigree. GALC enzyme activity detection and gene analysis were performed to confirm the diagnosis. Moreover, we reviewed all studies available on PubMed to understand the correlationship between phenotype and genotype of the identified mutations.

Results: The proband presented with sudden-onset weakness of left limbs with selective pyramidal tract involvement on diffusion-weighted imaging (DWI) of brain MRI. The GALC enzyme activity of him was low, and the GALC gene analysis revealed compound heterozygous pathogenic mutations of c.1901T $>C$ and c.1901delT. More interestingly, the homozygous c.1901T>C mutations were found in the proband's asymptomatic father and two paternal uncles. Meanwhile, the literature review revealed the c.1901T $>C$ mutation was only found in the late-onset form of $\mathrm{KD}$.
\end{abstract}

Conclusions: These observations, combined with previous reports, indicate that KD should be considered in the adult patients presenting selective pyramidal tract impairment even with sudden onset.

Keywords: Krabbe disease (KD); GALC; genotype; phenotype; pyramidal tract

Submitted Mar 15, 2018. Accepted for publication Apr 04, 2018.

doi: $10.21037 /$ atm.2018.04.30

View this article at: http://dx.doi.org/10.21037/atm.2018.04.30

\section{Introduction}

Krabbe disease (KD) (OMIM \#245200), also known as globoid cell leukodystrophy (GLD), is a rare autosomal recessive lysosomal storage disorder that is caused by deficiency of the lysosomal enzyme galactocerebrosidase (GALC) (1). A lack of this key enzyme leads to the accumulation of galactosylsphingosine or psychosine, which is neurotoxic to both the central and peripheral nervous system, resulting in central white matter and peripheral nerve demyelination (2). The clinical forms of this disease vary based on the age of onset and clinical course. The classical early infantile type is the most common manifestation. Onset may also occur in later infancy, in childhood, or even in adulthood. Although the clinical manifestations of adult-onset KD are heterogeneous, 
its clinical hallmarks include slowly progressive spastic paraplegia with or without sensorimotor neuropathy. Acute onset and progression is very rare (3).

The GALC gene is mapped to chromosome $14 \mathrm{q} 31$ (4) and contains 17 exons spanning approximately $60 \mathrm{~kb}$ of genomic DNA (5). To date, more than 120 mutations in the GALC gene have been reported worldwide (6). Although GALC mutations have been extensively identified, extremely rare findings from the Chinese population have been reported, and a clear relationship between various genotypes and phenotypes has not been fully elucidated.

Herein, we report a case of adult-onset KD from a Chinese pedigree that initially presented with acute hemiplegia. Brain MRI showed selective pyramidal tract involvement with restricted water diffusion on DWI and low GALC activity in leukocytes. Compound heterozygous mutations of c.1901delT and c.1901T >C were identified in the GALC gene, and both variants have been previously reported as disease-causing mutations $(7,8)$. In further family investigations, homozygous mutations for c.1901T >C were found in the patient's father and two paternal uncles. Interestingly, these relatives were in their fourth or fifth decade of life, but they remained asymptomatic to date. However, selective pyramidal tract involvement on brain MRI and low GALC activity were also identified in the father. We reviewed all studies available on PubMed to understand the correlation between phenotype and genotype of the identified mutations in the GALC gene.

\section{Methods}

\section{Subjects}

A 20-year-old Chinese boy experienced sudden onset weakness of the left limbs during sexual intercourse 1 month prior to admission, and the symptom progressed slowly over the following days. His parents were non-consanguineous. He was born after uncomplicated gestation and delivery. After birth, he was found to have an abdominal wall defect and had a neoplasty operation. One-and-a-half years later, he had a herniorrhaphy operation because of inguinal hernia. His developmental milestones were normal without toxic exposure. No drug or alcohol consumption was reported.

General physical examination showed no lymphadenopathy, normal chest and cardiovascular findings, no hepatomegaly or splenomegaly, and no skin lesions except for the scar beside his umbilicus because of the neoplasty operation.
On neurologic examination, he was alert, oriented and cooperative, and he had no difficulties with speech. He made full marks in the Mini-Mental State Examination (MMSE). Both visual acuity and fields were intact, and the funduscopic findings appeared normal. Motor examination showed mild left-sided brachiocrural hemiparesis with exaggerated deep tendon reflexes. Babinski signs and Hoffman reflexes were positive, and pes cavus was found on both sides. Sensation was normal to light touch and pinprick as were the sense of joint position and vibration sense.

Brain MRI disclosed hyperintensity along the bilateral pyramidal tracts from the centrum semiovale to the cervical segment of the spinal cord on T2-weighted images (T2WI) and fluid-attenuated inversion recovery (FLAIR) images. No gadolinium enhancement was observed in the lesions. The pyramidal tract showed restricted water diffusion on diffusion-weighted imaging (DWI) (Figure 1).

Hematological studies, including a complete blood count, coagulation studies, serum electrolytes, systemic autoimmune antibodies, thyroid function, detection of heavy metals and TORCH, were unremarkable. CSF was acellular with a slightly elevated protein level $(0.54 \mathrm{~g} / \mathrm{L}$, normal range $<0.41 \mathrm{~g} / \mathrm{L})$, and the oligoclonal bands were negative. Additional testing, including blood amino acid testing, was ordered, which revealed mildly elevated alanine $(3,080.38$, normal range $250-2,500)$ and proline (497.28, normal range 50-400) levels. Nerve conduction studies and electromyography (EMG) were normal. Muscle biopsy revealed mild myogenic changes. GALC activity of leukocytes was $1.3 \mathrm{nmol} / \mathrm{g} / \mathrm{min}$ (control range $>17.2 \mathrm{nmol} / \mathrm{g} / \mathrm{min}$ ), which is consistent with a diagnosis of $\mathrm{KD}$.

The parents, two paternal uncles, one aunt and grandparents of the proband were all in good health. None of the relatives had focal signs in neurological examination. The father's GALC activity of leukocytes was $3.5 \mathrm{nmol} / \mathrm{g} / \mathrm{min}$ (normal range $>17.2 \mathrm{nmol} / \mathrm{g} / \mathrm{min}$ ), and brain MRI revealed hyperintensities of the bilateral pyramidal tracts at the centrum semiovale level. Visual radiations were also involved (Figure 1). Thus, the patient, despite having GALC deficiency and characteristic MRI lesions, was asymptomatic. The other family members refused to have further examinations except for genetic testing.

All subjects provided written informed consent for the genetic studies and their publication.

\section{Sanger sequencing}

Genomic DNA-based PCR amplification and sequence 

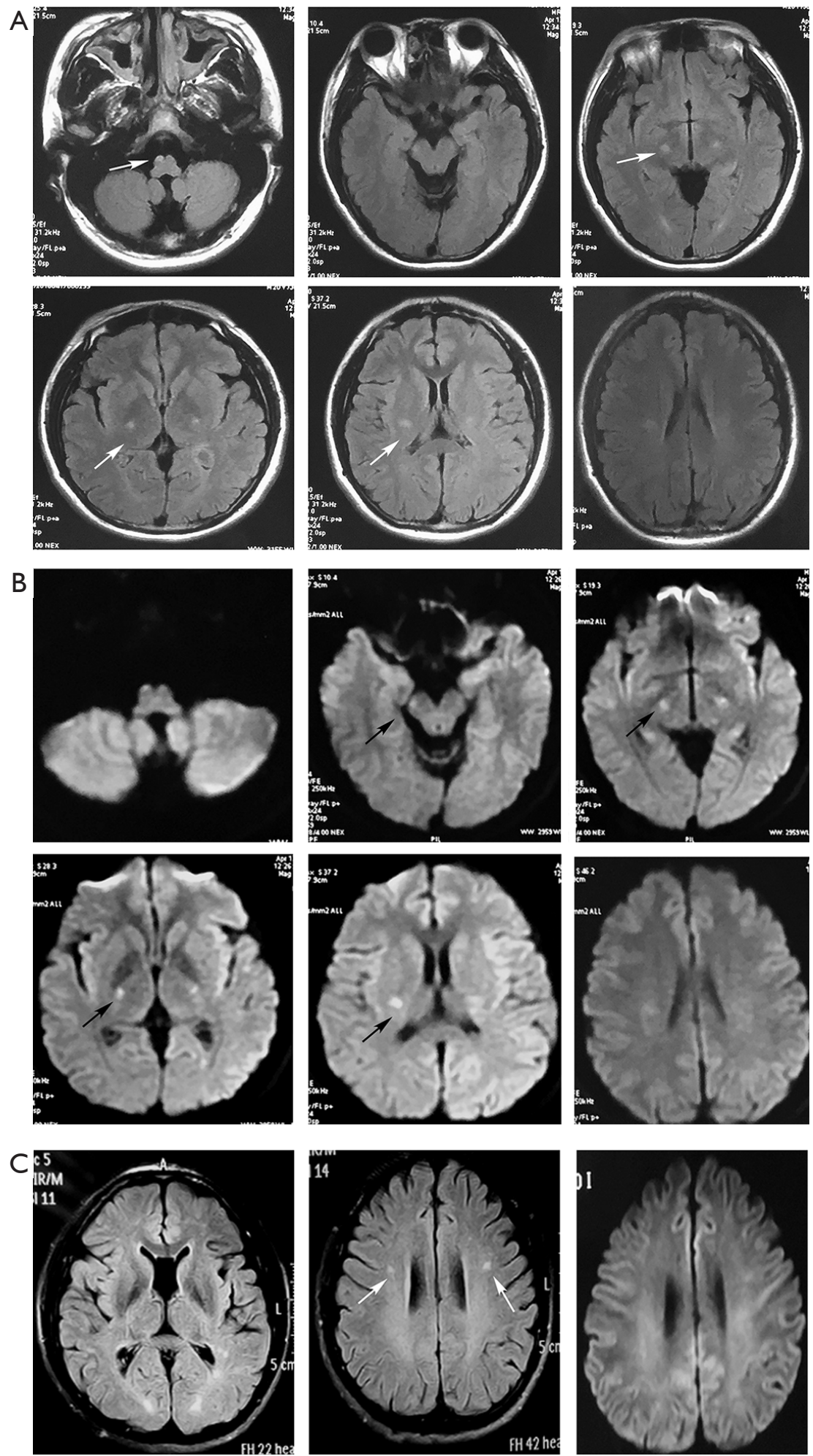

Figure $1 \mathrm{MRI}$ studies of the proband (A,B) and his father (C). (A) Axial fluid-attenuated inversion recovery (FLAIR) images of the proband exhibit hyperintensity along the bilateral pyramidal tracts; (B) corresponding planes of diffusion-weighted imaging (DWI) showed diffusion restricted lesions; (C) hyperintensities of the bilateral pyramidal tracts and visual radiations were found on the FLAIR images of the father, but no restricted water diffusion was detected on DWI. 
analysis of the GALC gene was performed. Genomic DNA was extracted from peripheral blood leukocytes of the proband and his family members. Control DNA from unaffected Chinese individuals was also obtained from peripheral blood leukocytes (samples were provided by the Institute of Medical Genetics, Shandong University). PCR amplifications of all coding regions and intron-exon boundaries of the GALC gene were carried out under standard conditions, and PCR products were subjected to direct bidirectional sequencing. All references to nucleotides or amino acids were based on the cDNA reference sequence NM_000153 for the GALC gene.

\section{Mutation nomenclature}

Following the HGVS guidelines is recommended for mutation nomenclature; these guidelines suggest using the first ATG as a translation initiation codon at nucleotide +1 (http://www.HGVS.org/varnomen). However, GALC mutations have been previously reported with the second ATG as nucleotide $+1(9,10)$. Hence, throughout this article, we ascribe HGVS designations to specific amino acid residues with the traditional designations given in parentheses.

\section{Results}

Molecular analysis showed compound heterozygous mutations of c.1901T>C (c.1853T>C) and c.1901delT (c.1853delT) in the proband, which would result in the p.L634S (p.L618S) amino acid transition and stop codon p.L634X (p.L618X), respectively. Familial segregation of the mutation of genomic DNA disclosed a paternal origin of c.1901T>C $(c .1853 \mathrm{~T}>\mathrm{C})$ and a maternal origin of c.1901delT (c.1853delT). To our surprise, the father was confirmed to be homozygous for c.1901T>C (c.1853T>C) mutation, as were the two paternal uncles. Then, the grandparents were confirmed to be carriers of this point mutation; they coincidently passed the mutation to their respective sons (Figure 2).

\section{Discussion}

Although the clinical manifestation of adult-onset KD is heterogeneous, involvement of the pyramidal tract is characteristic with an incidence as high as $82-94 \%(3,11,12)$. The MRI findings in the present case are consistent with previous reports. In addition, the clinical course of adultonset $\mathrm{KD}$ is considered to be mostly chronic and slowly progressive. Acute progression is very rare. There have
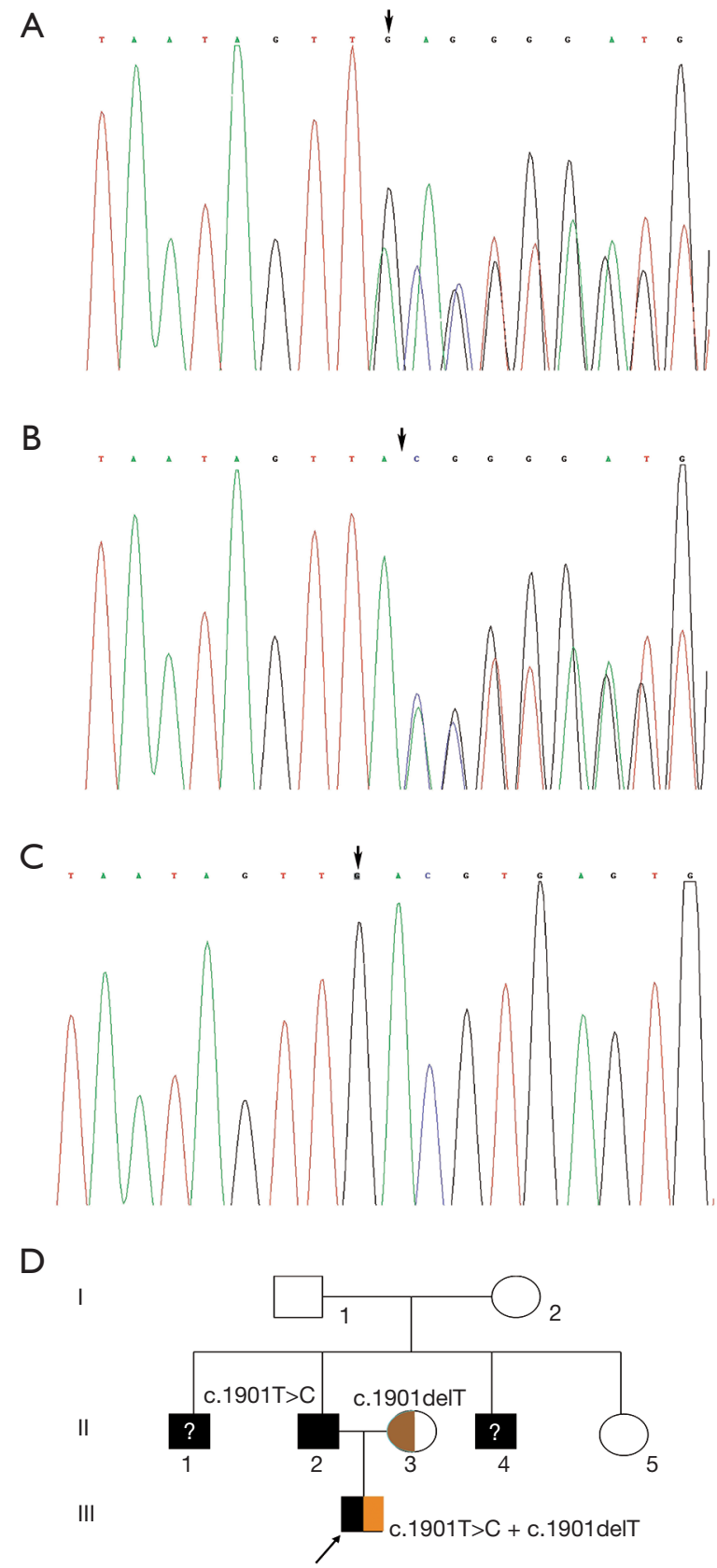

Figure 2 Genomic analysis (A,B,C) and pedigree chart (D) of the family. (A) GALC gene sequencing confirmed the compound c.1901delT and c.1901T >C mutations in the proband; (B) the heterozygous carrier state of c.1901delT in the mother; (C) the homozygous mutation of c.1901T >C in the father and two paternal uncles; (D) the pedigree chart of the family, where the proband is indicated by an arrow and the affected individuals are shown as closed symbols. The family members' number are listed as 1-5.The two paternal uncles (II-1 and II-4) are shown as "?" because they were theoretically preclinical patients, but no additional clinical evidence was available. 
Table 1 Genotype-phenotype correlation for p.L634S (p.L618S) mutation in previously reported KD patients

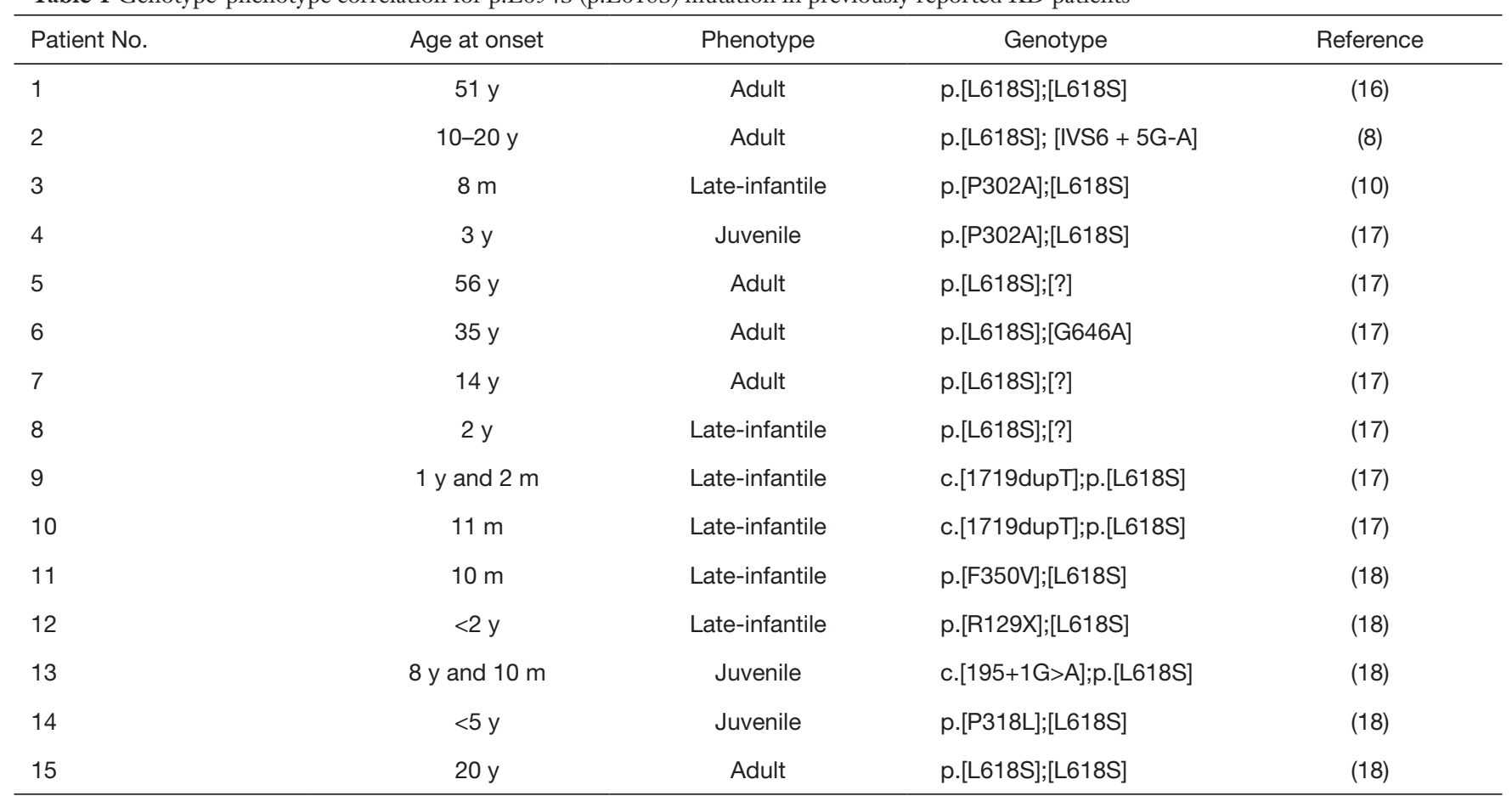

$\mathrm{KD}$, Krabbe disease; $y$, year; $\mathrm{m}$, month.

been only three reported cases of acute exacerbation similar to the present one (13-15). Meanwhile, only one report (15) mentioned a case of $\mathrm{KD}$ with restricted diffusion on DWI corresponding to exacerbation of the condition. In our case, restricted diffusion on DWI consistent with acute progression was also found in the proband but not in the asymptomatic father. Therefore, it seems that DWI is helpful in the follow-up of $\mathrm{KD}$ to assess disease progression.

In addition, whether MRI abnormalities occurred before symptoms is a debated issue (12). In our study, scans were analyzed not only for the symptomatic proband but also for the asymptomatic father, which indicates that MRI abnormalities may present before symptom onset. If MRI abnormalities precede symptom onset, the ability of MRI to predict phenotypes in newborn screening populations would be greatly enhanced, and effective therapeutic intervention in asymptomatic patients with late-onset phenotypes may be possible at an early stage of the disease.

In the present study, we identified compound heterozygous mutations in the GALC gene of the proband. The mutation c.1901delT (c.1853delT) had been found in a Mexican patient only (7). The other mutation, c.1901T >C (c.1853T >C), was first reported in a Japanese patient with late onset
$\mathrm{KD}(8)$ and then described in many late-onset patients (16-18). We reviewed all studies related to $c .1901 \mathrm{~T}>\mathrm{C}$ (c.1853T>C) or p.L634S (p.L618S) that were available on the PubMed and listed the clinical phenotypes in Table 1. This mutation has been reported in Japanese and Chinese populations and contributed to the late-onset form only. As we know, autosomal recessive diseases with the onset of two generations in one family are very rare, but if they are found, it is important to elucidate the genotype-phenotype correlation of the mutation. Therefore, we presented the phenotype of the c.1901T>C $(c .1853 \mathrm{~T}>\mathrm{C})$ mutation in one Chinese family in both heterozygous and homozygous states and confirmed the previous conclusion that all phenotypes of this mutation were the late-onset form. The phenotype of the proband with compound heterogeneous mutations is more serious than that of the homozygous father, which is attributed to the amino acid changes of these two mutations. The nonsense mutation c.1901delT (c.1853delT), which results in a stop codon, seems to be functionally important since polypeptide elongation was stopped at the 634th amino acid because of the premature stop codon; this mutation caused a larger structural change than the point mutation c.1901T>C (c.1853T>C). 


\section{Conclusions}

The presented pedigree in our study verified the genotypephenotype relation of $\mathrm{KD}$ in two generations of a Chinese pedigree. We emphasize that KD should be included in differential diagnosis of selective pyramidal tract impairment in adults even if patients with an abrupt onset. If diffusion restriction is found on DWI, it may predict an acute exacerbation of KD. In addition, the c.1901T>C (c.1853T>C) mutation in the GALC gene will lead to a mild phenotype, and a patient harboring this mutation, especially the homozygous type, may have a better prognosis.

\section{Acknowledgments}

We thank the patient and his family for their participation. Funding: The study was supported by grants from the Fundamental Research Fund of Shandong University (No. 2014QLKY27) and the Promotive Research Fund for Young and Middle-Aged Scientists of Shandong Province, China (No. BS10YY026).

\section{Footnote}

Conflicts of Interest: The authors have no conflicts of interest to declare.

Ethical Statement: The present study was approved by the Institute Ethical Committee of Qilu Hospital of Shandong University, Jinan, China.

\section{References}

1. Suzuki K, Suzuki Y. Globoid cell leucodystrophy (Krabbe's disease):deficiency of galactocerebroside betagalactosidase. Proc Natl Acad Sci U S A 1970;66:302-9.

2. Svennerholm L, Vanier MT, Månsson JE. Krabbe disease: a galactosylsphingosine (psychosine) lipidosis. J Lipid Res1980;21:53-64.

3. Debs R, Froissart R, Aubourg P, et al. Krabbe disease in adults: phenotypic and genotypic update from a series of 11 cases and a review. J Inherit Metab Dis 2013;36:859-68.

4. Cannizzaro LA, Chen YQ, Rafi MA, et al. Regional mapping of the human galactocerebrosidase gene (GALC) to $14 \mathrm{q} 31$ by in situ hybridization. Cytogenet Cell Genet 1994;66:244-5.

5. Luzi P, Rafi MA, Wenger DA. Structure and organization of the human galactocerebrosidase (GALC) gene. Genomics 1995;26:407-9.

6. Graziano AC, Cardile V. History, genetic, and recent advances on Krabbe disease. Gene 2015;555:2-13.

7. Wenger DA, Rafi M, Luzi P. Molecular genetics of Krabbe disease (globoid cell leukodystrophy): diagnostic and clinical implications. Hum Mutat 1997;10:268-79.

8. Furuya H, Kukita Y, Nagano S, et al. Adult onset globoid cell leukodystrophy (Krabbe disease):analysis of galactosylceramidase cDNA from four Japanese patients. Hum Genet 1997;100:450-6.

9. Wenger DA, Rafi MA, Luzi P, et al. Krabbe disease: genetic aspects and progress toward therapy. Mol Genet Metab 2000;70:1-9.

10. Xu C, Sakai N, Taniike M, et al. Six novel mutations detected in the GALC gene in 17 Japanese patients with Krabbe disease and new genotype-phenotype correlation. J Hum Genet 2006;51:548-54.

11. Farina L, Bizzi A, Finocchiaro G, et al. MR imaging and proton MR spectroscopy in adult Krabbe disease. AJNR Am J Neuroradiol 2000;21:1478-82.

12. Abdelhalim AN, Alberico RA, Barczykowski AL, et al. Patterns of magnetic resonance imaging abnormalities in symptomatic patients with Krabbe disease correspond to phenotype. Pediatr Neurol 2014;50:127-34.

13. Tomás J, Durães J, Lacerda L, et al. Adolescent-onset Krabbe disease with an initial diagnosis of multiple sclerosis and a novel mutation. BMJ Case Rep 2015;2015.

14. Arenson NE, Heydemann PT. Late-onset Krabbe's disease mimicking acute disseminated encephalomyelitis. Pediatr Neurol 2005;33:208-10.

15. Mamada N, Nakamagoe K, Shioya A, et al. Adult-onset Krabbe disease presenting as acute hemiparesis and progressive demyelination detected by diffusion- weighted imaging. J Neurol Sci 2016;367:326-8.

16. Satoh JI, Tokumoto H, Kurohara K, et al. Adult-onset Krabbe disease with homozygous T1853C mutation in the galactocerebrosidase gene. Unusual MRI findings of corticospinal tract demyelination. Neurology 1997;49:1392-9.

17. Hossain MA, Otomo T, Saito S, et al. Late-onset Krabbe disease is predominant in Japan and its mutant precursor protein undergoes more effective processing than the infantile-onset form. Gene 2014;534:144-54.

18. Zhao S, Zhan X, Wang Y, et al. Large-scale Study of Clinical and Biochemical Characteristics of Chinese Patients Diagnosed with Krabbe Disease. Clin Genet 2018;93:248-54.

Cite this article as: Zhang T, Yan C, Ji K, Lin P, Chi L, Zhao $\mathrm{X}$, Zhao Y. Adult-onset Krabbe disease in two generations of a Chinese family. Ann Transl Med 2018;6(10):174. doi: 10.21037/ atm.2018.04.30 